\title{
Prediction for Outlet Noise of Rolling Piston Comperssor
}

\author{
Tieshan Zhang ${ }^{1, a}$ \\ ${ }^{1}$ Institute of Vibration and Noise Control, School of Mechanical Engineering \\ Xi'an Jiaotong University, Xi'an, China
}

\begin{abstract}
An acoustic wave equation with considering small perturbation is presented first by use of the fluidic mechanics and aerodynamics, then a theoretical model for predicting the outlet noise of rolling piston compressors is investigated, and the sound pressure and sound power of the outlet noise are formulated based on the acoustic wave equation. The experimental data and simulation results for the outlet noise with different rotation velocities have been compared with the discrepancy less than $2.6 \%$, which verifies the approach presented in this paper.
\end{abstract}

\section{Introduction}

Many works concerned with the noise control of compressors have been published up to now, which were mainly focused on the noise and vibration control of rolling piston compressors, especially on the control of the structural noise and outlet noise of a whole compressor (1, 2, 3, 4 and 5). However, there are few literatures on the noise mechanism and the affection factors. Therefore, a theoretical model is presented to predict the outlet noise of rolling piston compressors, which is also verified by the experimental measurement with different rotation velocities. Based on this, an approach to reduce the outlet noise has been put forward, and would provide a theoretical direction for designing low noise compressors.

\section{Theoretical model of outlet noise}

In general, the outlet valve will be moving quickly under the impulse of air stream when the compressor is working. Accordingly, the resulting disturbance in the ambient media will produce the sound pressures in practice.

\subsection{Theoretical modeling}

Considering an infinite duct, a thin film devides the static air in the duct into two parts, as shown in Fig1 $(6,7)$. Their pressures are $\mathrm{P} 1$ and $\mathrm{P} 2(\mathrm{P} 2>\mathrm{P} 1), \quad(\mathrm{P} 2-$ $\mathrm{P} 1=\Delta \mathrm{P}$. Assuming that $\Delta \mathrm{P}(\mathrm{P} 2-\mathrm{P} 1=\Delta \mathrm{P})$ is very small, the temperatures of the two parts are identical and the film is located on the origin of the coordinates. Provided that the film disappeared at some time suddenly, a disturbance wave would arise from the high pressure part to the low pressure segment and increase the pressure of the low pressure part. Likewise, another disturbance

\footnotetext{
a Corresponding author: author@e-mail.org
}

wave would arise simultaneously from the low pressure part to the high pressure one and decrease the pressure of the high pressure part. Because the pressure difference of the two parts is a small quantity, the disturbance waves are also the small quantity waves that propagate in the sound velocity. In view of the assumption of the temperature in the two parts, the sound velocities of the two waves will be the same. There is a disturbance field in the vicinity of the film location. Apart from the field, the media will keep the initial status before the wavefronts reach. As shown in Fig.1, the control volume includes two wavefronts, where $\mathrm{c}$ is the sound velocity, $\rho_{0}$ is the material density of air and P denotes the sound pressure.

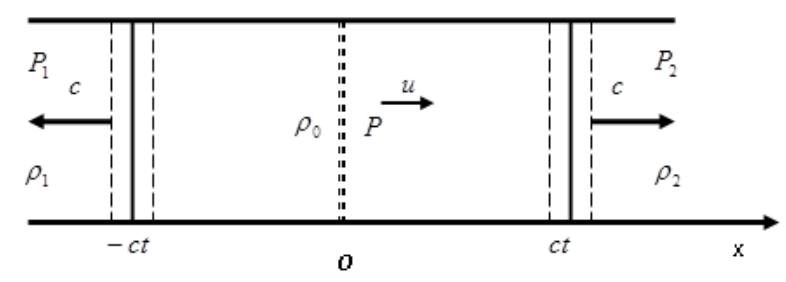

Figure 1. The little disturbance disseminating in two directions.

For the wavefront $x=-c t$, the air velocity of the left is $\mathrm{c}$ and that of the right is $c+u$. According to the mass and energy conservation of the control volume, it can be obtained that:

$$
\begin{gathered}
\rho_{1} c=\rho_{0}(c+u), \\
P_{1}+\rho_{1} c^{2}=P+\rho_{0}(c+u)^{2}
\end{gathered}
$$

where $\mathrm{u}$ is the particle velocity of air.

Combing Eq.(1) and Eq.(2), the pressure of air can be given

$$
P=P_{1}-\rho_{1} c u
$$


Similarly, for the wavefront $x=c t$, the relations can be also derived as follows

$$
\begin{gathered}
\rho_{2} c=\rho_{0}(c-u), \\
P_{2}+\rho_{2} c^{2}=P+\rho_{0}(c-u)^{2}, \\
P=P_{2}+\rho_{2} c u .
\end{gathered}
$$

Replacing Eq.(3) into Eq.(6) yields

$$
P_{2}-P_{1}=\Delta P=-\left(\rho_{1}+\rho_{2}\right) c u
$$

From Eq.(7), the particle velocity can be expressed as

$$
u=-\frac{P_{2}-P_{1}}{\left(\rho_{1}+\rho_{2}\right) c}
$$

Further, according to Eqs.(1) and (4), one can obtain

$$
\rho_{0}=\frac{1}{2}\left(\rho_{1}+\rho_{2}\right)
$$

Substituting Eq.(9) into Eq.(8), the particle velocity can be written as

$$
u=-\frac{\Delta P}{2 c \rho_{0}},
$$

where the minus means that the particle velocity is opposite to the positive $\mathrm{x}$ axis.

Because the difference of the air pressure in the two parts is small quantity, the disturbance waves will disseminate in the two directions, and the disturbance velocity $\mathrm{u}$ in the media is also very small.

\subsection{Exhaust procedure of rolling piston compressor}

on the basis of the work principle of rolling piston compressors (8), their exhaust procedure can be approximated as the equivalent model, as shown in Fig.2. When air in the exhaust duct is compressed, the air pressure in the duct will increase in a short time. Then the air pressure in the exhaust duct will be gradually equal to the external force acted on the valve. If the piston squeezes the air in the duct continually and the resulting air pressure is larger than the external force on the valve, the valve of the compressor will be opened and the exhaust process will be started. The enduring time would be much shorter despite the compressibility of air. That is to say, the opening of the valve is finished instantaneously. In consequence, the air pressure disturbance in the duct caused by the valve opening features the instant disturbance and the procedure could be formulated by the above theoretic mode.

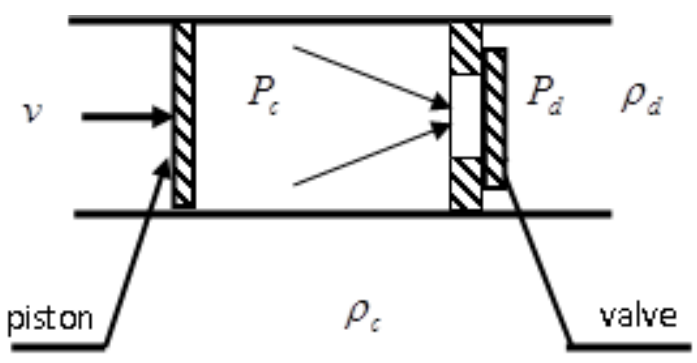

Figure 2. Schematic of work principle of rolling piston compressor.

\section{Radiating sound energy prediction of exhaust noise}

According to the presented theoretic model, we could predict the sound energy of the exhaust noise. Meanwhile, the factors that affect the exhaust noise of compressors will be proposed.

\subsection{Formulation of radiating sound energy}

The exhaust noise could be modeled as a point sound resource (9) when the compressor is on exhaust, according to the exhaust characteristics and the structure of the compressor (1). The idealization of the sound source would avoid the tedious mathematic computation and the results could exhibit the fundamental principle.

\subsubsection{Sound radiation of sphere resource}

Consider a sphere whose radius is ${ }^{r_{0}}$, as depicted in Fig.3. The surface of the sphere varies harmonically in small quantity $\xi=d r$. Therefore, the spherical wave will be present and spread in the media. Assuming that the origin of the coordinates is located at the center of the sphere, the wave equation can be given by Eq.(11), where $\mathrm{S}$ is the surface area of the sphere and $S=4 \pi r^{2}$. Accordingly, the wave equation can be rewritten as

$$
\begin{gathered}
\frac{\partial^{2} p}{\partial r^{2}}+\frac{\partial p}{\partial r} \frac{\partial \ln S}{\partial r}=\frac{1}{c_{0}^{2}} \frac{\partial^{2} p}{\partial t^{2}} \\
\frac{\partial^{2} p}{\partial r^{2}}+\frac{2}{r} \frac{\partial p}{\partial r}=\frac{1}{c_{0}^{2}} \frac{\partial^{2} p}{\partial t^{2}}
\end{gathered}
$$

The solution of Eq.(12) can be given by

$$
p=\frac{A}{r} e^{j(\omega t-k r)}+\frac{B}{r} e^{j(\omega t+k r)}
$$


Considering there is no reflection wave, the second term of the solution will be ignored, i.e. $B=0$. So the final solution can be obtained

$$
p=\frac{A}{r} e^{j(\omega t-k r)},
$$

where the constant A maybe complex number, the absolute value of $A / r$ is just the amplitude of the sound pressure. For the one-dimensional wave equation of small amplitudes, the particle velocity $v$ and the sound pressure $^{p}$ hold the relation

$$
\rho_{0} \frac{\partial v}{\partial t}=-\frac{\partial p}{\partial x}
$$

Next, the particle velocity in the radius direction can be given by

$$
v_{r}=-\frac{1}{j \omega \rho_{0}} \frac{\partial p}{\partial r}=\frac{A}{r \rho_{0} c_{0}}\left(1+\frac{1}{j k r}\right) e^{j(\omega t-k r)}
$$

Note that Eqs.(14) and (16) represent the general form of the radiating sound field for the pulse sphere sound source.

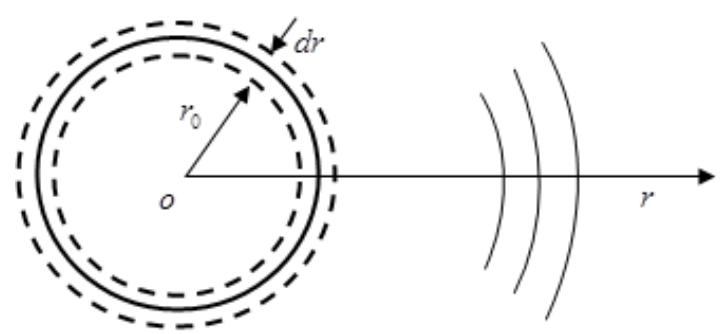

Figure 3. Sound radiation from pulse sphere.

Provided that the vibration velocity of the sphere surface is $\mathrm{u}$, the particle velocity on the sphere surface will be identical to the vibration velocity of the sphere surface. So the boundary condition can be expressed as

$$
\left(v_{r}\right)_{r=r_{0}}=u_{a} e^{j\left(\omega t-k r_{0}\right)} .
$$

Then combining Eqs.(16) and (17), the radiating sound pressure of the pulse sphere source can be obtained as

$$
p=p_{a} e^{j(\omega t-k r+\theta)}
$$

where $p_{a}=\frac{|A|}{r},|A|=\frac{\rho_{0} c_{0} k r_{0}^{2} u_{a}}{\sqrt{1+\left(k r_{0}\right)^{2}}}$, and $\quad \theta=\arctan \left(\frac{1}{k r_{0}}\right)$

Substituting A into Eq.(16), we could get the particle velocity of the radiating sound field for the pulse sphere source

$$
v_{r}=v_{r a} e^{j\left(\omega t-k r+\theta+\theta^{\prime}\right)}
$$

where $v_{r a}$ is the particle velocity amplitude in radius direction, $v_{r a}=p_{a} \frac{\sqrt{1+(k r)^{2}}}{\rho_{0} c_{0} k r}, \theta^{\prime}=\arctan \left(\frac{-1}{k r}\right)$.

Energy relation in spherical sound field

In the spherical acoustic field, the sound intensity is given by

$$
I=\frac{1}{T} \int_{0}^{T} \operatorname{Re} p \operatorname{Re} v_{r} d t
$$

Inserting Eqs.(18) and (19) into Eq.(20), the sound intensity can be shown as

$$
\begin{gathered}
I=\frac{1}{T} \int_{0}^{T} p_{a}^{2} \frac{\sqrt{1+(k r)^{2}}}{\rho_{0} c_{0} k r} \cos (\omega t-k r+\theta) \cos \left(\omega t-k r+\theta+\theta^{\prime}\right) d t \\
=p_{a}^{2} \frac{\sqrt{1+(k r)^{2}}}{\rho_{0} c_{0} k r} \frac{\cos \theta^{\prime}}{2} .
\end{gathered}
$$

According to the relation $\cos \theta^{\prime}=\frac{k r}{\sqrt{1+(k r)^{2}}}$, the sound intensity is rewritten as

$$
I=\frac{p_{a}^{2}}{2 \rho_{0} c_{0}}
$$

Subsequently, the average sound power through the sphere surface whose radius is equal to $r$ can be expressed as

$$
\bar{W}=4 \pi r^{2} I=2 \pi r^{2} \frac{p_{a}^{2}}{\rho_{0} c_{0}}=\frac{2 \pi}{\rho_{0} c_{0}}|A|^{2}
$$

\subsubsection{Theoretic model of exhaust noise}

In the study, the radius of the exhaust noise source of the rolling piston compressor is $0.0055 \mathrm{~m}$. The main peak frequencies of the exhaust noise range from $250 \mathrm{~Hz}$ to $6.3 \mathrm{k} \mathrm{Hz}$. And the maximum operating frequency of the compressor is $180 \mathrm{~Hz}$. That is to say, the condition $k r_{0}<<1$ is satisfactory to the compressor in the work. Further, in terms of the exhaust structure characteristics of the compressor, the source of the exhaust noise could be idealized as the model as shown in Fig.4. the sound pressure can be approximated as

$$
p \approx j \frac{k \rho_{0} c_{0}}{2 \pi r} Q_{0} e^{j(\omega t-k r)}
$$

where $Q_{0}=2 \pi r_{0}^{2} u_{a}$.

From the above theory, the variable $u$ represents the particle velocity while the thin film disappeared. Here the variable $u$ can be regarded as the particle velocity of the exhaust noise while the exhaust valve is opening instantaneously. 


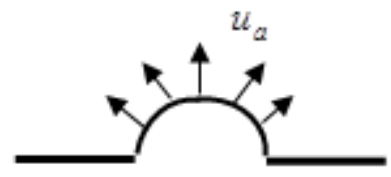

Figure 4. Theoretical model of the sound radiation from exhaust noise.

\section{Force on exhaust valve analysis}

Under the practical operation, the forces applied on the exhaust valve are depicted in Fig.5, where $P_{c}$ is the air pressure in the exhaust cavity, $P_{d}$ is the air pressure out of the exhaust cavity, $P_{\mu}$ is the viscous force caused by the oil film between the valve and valve seat when the valve is opening, $P_{m}$ is the inertial force of the moving valve, and $P_{e}$ is the elastic force of the valve.

In the above formulation, the inertial force of air is not considered since the air amount released is much less at the opening time of the valve. As a consequence, the inertial force of air will be much less and ignored.

From Fig.5, when the compressor is exhausting, the equilibrium equation of forces applied on the valve can be given by

$P_{c} \frac{\pi}{4} d_{1}^{2}=P_{d} \frac{\pi}{4} d_{2}^{2}+P_{m}+P_{\mu}+P_{e}$

where $d_{1}$ is the diameter of the exhaust hole, and $d_{2}$ is the diameter of the valve

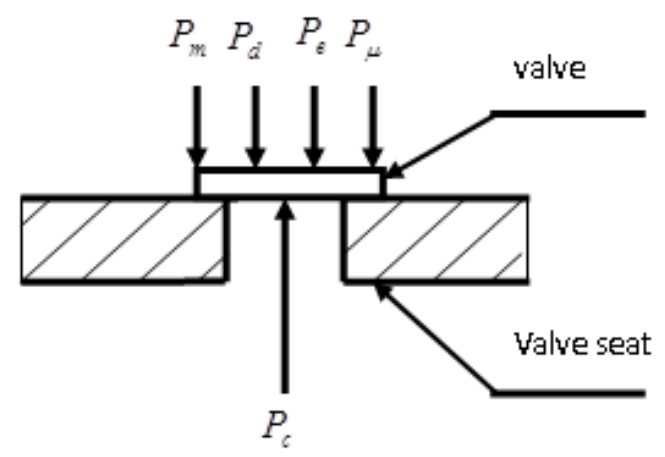

Figure 5. Schematic of the forces on exhaust valve

Let $d_{2}=d_{1}+\Delta d$, from Eq.( 25), one can obtain

$P_{c} \frac{\pi}{4} d_{1}^{2}+P_{d} \frac{\pi}{4}\left(d_{1}^{2}+2 d_{1} \Delta d+\Delta d^{2}\right)=P_{m}+P_{e}+P_{\mu}$

Next, the small quantity of the first order and the second order can be ignored, one can get

$$
P_{c}-P_{d}=\frac{4}{\pi d_{1}^{2}}\left(P_{m}+P_{e}+P_{\mu}\right)=\Delta P
$$

In Eq.(26), $\left(P_{c}-P_{d}\right)$ is the difference of the disturbance pressure. According to Eq.(8), $\left(P_{2}-P\right)_{1}$ is $\Delta P$, i.e. $\left(P_{c}-P_{d}\right)$. Considering the direction of the air flow, Eq.(8) is rewritten as

$$
u=\frac{P_{2}-P_{1}}{\left(\rho_{2}+\rho_{1}\right) c}
$$

Substituting Eq.(26) into Eq.(27), one obtains

$$
u=\frac{P_{m}+P_{\mu}+P_{e}}{\left(\rho_{2}+\rho_{1}\right) c}
$$

Moreover, combining Eqs.(28) and (24), the sound pressure can be given by

$$
p \approx j \frac{k \rho_{0} c_{0}}{2 \pi r} Q_{0} e^{j(\omega t-k r)}
$$$$
Q_{0}=2 \pi r_{0}^{2} \frac{\Delta P}{2 c \rho_{0}}
$$

According to the relation of $c=c_{0}$, Eq.(29) can be rewritten the form

$$
p \approx j \frac{k r_{0}^{2}}{2 r} \frac{4}{\pi d_{1}^{2}}\left(P_{m}+P_{\mu}+P_{e}\right) e^{j(\omega t-k r)}
$$

and the sound pressure level of the exhaust noise can be expressed as

$\mathrm{SPL}=20 \log p / p_{0}=20 \log \left(\frac{j \frac{k r_{0}^{2}}{2 r} \frac{4}{\pi d_{1}^{2}}\left(P_{m}+P_{\mu}+P_{e}\right) e^{j(\omega t-k r)}}{p_{0}}\right)$

where $p_{0}$ is the reference sound pressure, $p_{0}=10^{-5} \mathrm{~Pa}$. The unit of SPL is $\mathrm{dB}$. Let the relation $r_{0}=d_{1} / 2$ into Eq.(31) yields

$\mathrm{SPL}=20 \log \left[j \frac{f}{c r p_{0}}\left(P_{m}+P_{e}+P_{\mu}\right) e^{j(\omega t-k r)}\right]$.

In Eq.(32), when the operating frequency keeps constant, it can be found that the sound pressure level of the sound radiation from the compressor is just proportional to the inertial force, the elastic force and the viscous force of the exhaust valve.

\section{Numerical results}

In this section, we will give the numerical examples according to the method established previously. The parameters of the theoretic model in the numerical computation are as follows: the position of the 
measurement point $r=0.3 \mathrm{~m}$; the sound speed $c=340 \mathrm{~m} / \mathrm{s}$; the operating frequency $f=50 \mathrm{~Hz}$; the inertial force of the valve $P_{m}=1.5 \mathrm{~N}$; the elastic force of the valve $P_{e}=1 \mathrm{~N}$; the viscous force between the valve and seat $P_{\mu}=0.5 \mathrm{~N}$.

Fig. 6 shows the sound pressure level of the exhaust noise in a period. It can be seen that the maximum value of the sound pressure level is $97.32 \mathrm{~dB}$. In terms of the identical parameters, the measurement datum is $96.10 \mathrm{~dB}$ in the laboratory. That is to say, the discrepancy between the experimental value and numerical result is $1.2 \%$ under the same configuration

\section{Experimental verification}

From Eq.(32), it is noticeable that the factors affecting the sound pressure level of the compressor include the operating frequency, the elastic force, the inertial force and viscous force, etc. For the purpose of validating the formulation, the experiment is conducted to measure the radiating noise under various frequencies.

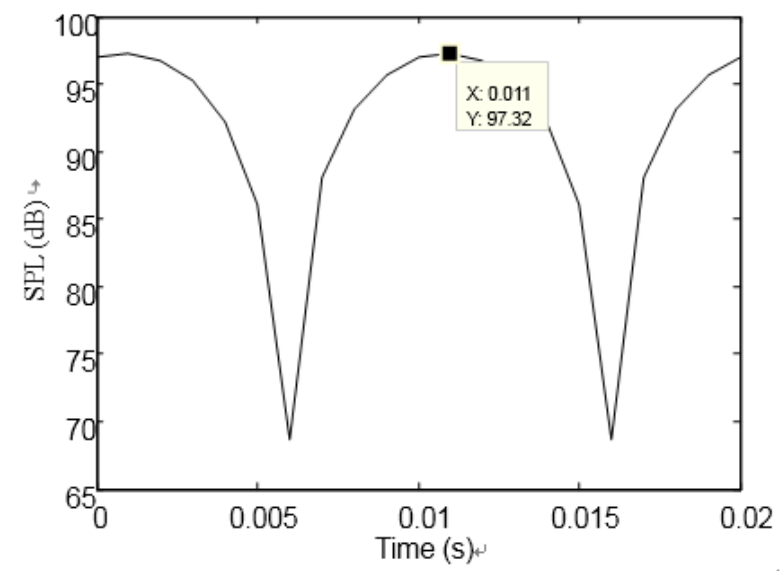

Figure 6. Sound pressure level of radiating noise.

Fig.7 plots the sound pressure level of the exhaust noise with the different rotating speed for the bear compressor. It is noted that the exhaust noise will increase with the operating frequency increasing. Further, the relation of the sound pressure level of the exhaust noise and the rotating speed is linear approximately. In consequence, the exhaust noise of compressors is a kind of special noise source rather than the common ones, and owns the particular acoustic mechanism.

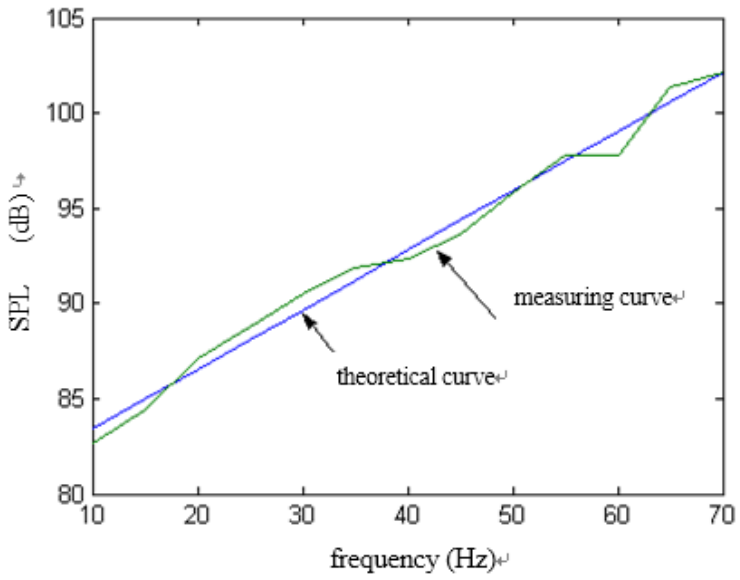

Figure 7. Exhaust noise versus rotating frequency

Fig.8 illustrates the theoretic value and the experimental data of the exhaust noise, and it suggests that trends of the two change curves is similar basically. When $x=65 \mathrm{~Hz}$, the extreme difference of the two curves is near $2.6 \%$. To sum up, the theoretic prediction of the exhaust noise is very close to the experimental data, and the presented method is valuable in the practical engineering.

\section{Conclusion}

The theoretic model to depict the sound radiation of the exhaust noise for rolling piston compressors is investigated in this paper. The sound prediction of the exhaust noise is formulated by using the method presented, and the numerical simulation is implemented. Meanwhile, the characteristics and generating mechanism of the exhaust noise is addressed according to the theoretic model. Further, the validity of the

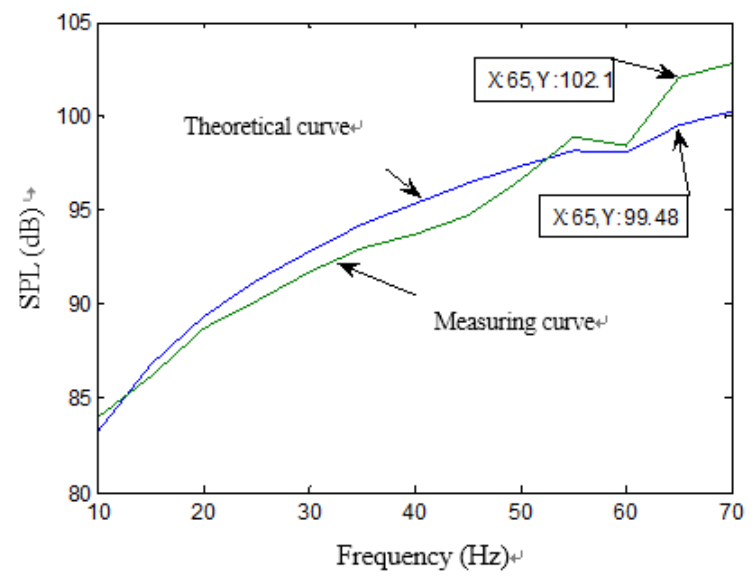

Figure 8. Comparison of theoretical value and experimental result.

method is verified by the physical experiment. The maximum difference between the prediction values and the experimental results is $2.6 \%$. In conclusion, the main factors influencing the sound radiation of the exhaust noise consist of the rotating frequency of compressors, the elastic force of the valve, the inertial force of the valve and the viscous force between the valve and the 
valve seat. Moreover, the sound pressure level of the radiating noise and the rotating frequency feature the approximate linear relation.

\section{Acknowledgments}

The work described in this paper was supported by Program for New Century Excellent Talents in University of China (Approval No. NCET-06-0842).

\section{References}

1. Ma YC, Min OK. Pressure calculation in a compressor cylinder by a modified new helmholts modelling. Jounal of Sound and Vibration, 2001, 243(5):775-796.

2. Lee JH, Kim J. Sound transmission through cylindrical shell of hermetic compressor. Proceedings of the 2000 international compressor engineering conference at Purdue, Purdue University Press, 2000, 933-940.

3. Suh KH. The analysis of the discharge muffler in the rotary compressors. Proceedings of the 2000 international compressor engineering conference at Purdue, 2000, 651-656.

4. Cui MM. Unsteady flow around suction elbow and inlet guide vanes in a centrifugal compressor, Proceedings of the Institution of Mechanical Engineers, Part G: Journal of Aerospace Engineering, 2006, 220(1), 11-28.

5. Park Jongchan, Wang Semyung. Noise reduction for compressors by modes control using topology optimization of eigenvalue. Journal of Sound and Vibration, 2008, 315(4-5): 836-848.

6. Luo Manlou. Aerodynamics. Shanghai Jiaotong University Press of China, 1989.

7. Zhuang Lixian, Yin Xieyuan, Luo Huiyang. Fluid mechanics. Shanghai Jiaotong University Press of China, 1991.

8. Ma Guoyuan, Li Hongqi. Rolling Piston Compressor. Chinese Mechanical Engineering Press, 2001.

9. Du Gonghuan, Zhu Zhemin, Gong Xiufen. Acoustics Basis. 2nd Ed., Nanjing University Press of China. 2001 . 